\title{
System Modelling of Mammalian Cell Cycle Regulation Using Multi-Level Hybrid Petri Nets
}

\author{
$\underline{\text { A. Abroudi }}^{\text {a }}$ and S. Samarasinghe ${ }^{\text {a }}$ \\ ${ }^{a}$ Integrated Systems Modelling Group, Department of Informatics and Enabling Technologies, \\ Lincoln University, Canterbury, New Zealand \\ Email: Ali.Abroudi@LincolnUni.ac.nz
}

\begin{abstract}
An important process in the growth of any biological organism is its ability to proliferate, a tightly controlled process in which a cell divides into two daughter cells. This happens within a dynamic environment, where a cell responds to various internal and external signals through a well-ordered sequence of events called cell cycle. Underlying these events are a complex and elegantly orchestrated web of interactions that function as an integrated system with various sub-systems that specialize in various tasks. The important task is the response of cell cycle machinery to proliferative signals in order to initiate a process which eventually leads to production of two daughter cells. Malfunction of cell cycle system causes diseases, such as cancer.

This study proposes a novel systems approach to modelling mammalian cell cycle to gain insights into how it coordinates such an intricate system of interactions in a robust and timely manner. It involves identification of the most essential controllers of mammalian cell cycle (as primary elements) and regulators of these controllers (as secondary elements) at different levels of abstraction to develop a minimal yet comprehensive model. In this paper, a Multi-Level Hybrid Petri Net (MLHPN), a graphical Petri Net-based modelling method, is proposed to model the mammalian cell cycle regulation system. Intuitive nature of MLHPN makes it possible to represent biological properties and processes with different time scales through a combination of continuous and discrete paradigms at different levels of abstraction. The goal is to gain a deep understanding of the mechanism of mammalian cell cycle regulation in the presence of growth factors.
\end{abstract}

Keywords: Computational systems biology, mammalian cell cycle regulation, Petri Nets (PNs), hybrid modelling 
Abroudi and Samarasinghe, System Modelling of Mammalian Cell Cycle Regulation Using Multi-Level Hybrid Petri Nets

\section{INTRODUCTION}

The cell reproduction is regulated through an accurate system of biochemical interactions called cell cycle during which a cell first grows, then its DNA becomes replicated, and eventually divides into two almost identical cells (daughter cells). A typical cell cycle comprises four ordered sequence of stages: G1, S, G2, and M phases (Morgan, 2007). A schematic of mammalian cell cycle is shown in Figure 1. During early G1 phase, if the environmental conditions favors the proliferation process (e.g. the presence of enough nutrients and growth factors), the cell leaves stationary state G0 and cell cycle machinery starts producing proteins required for cell cycle progression. This procedure leads to increase in cell size which is known to be the necessary condition for transition through Restriction Point (RP) in late G1. In fact, RP is a point beyond which a cell becomes independent of growth factors and committed to finishing the current cell cycle (Zetterberg et al., 1995). In contrast, if environmental conditions are not appropriate (before RP), the cell returns to G0 and cell cycle does not proceed. S phase is the second cell cycle phase in which DNA duplication takes place. After completion of S phase, the cell should make sure whether the duplication process has been appropriately completed or not. This occurs during G2 phase. Furthermore, proteins which are crucial for cell division are synthesized in G2 that results in further cell growth during this phase. The last phase (M phase) includes some sub-phases (Prophase, Metaphase, Anaphase, Telophase) in which chromatins become condensed to form chromosomes, nuclear envelop begins to dissolve, chromosomes are aligned on the spindle plate, sister chromatids are segregated, two nuclear membranes are reestablished and finally, during a stage called Cytokinesis, the cell becomes divided (Berridge, 2012). As can be seen in Figure 1, two DNA damage checkpoints also exist in cell cycle (G1-S and G2-M checkpoints) whose main role is to check DNA for any possible damage. And in the case of damage, they arrest the cell cycle progression to provide enough time for cell cycle machinery to repair the damage (if it is repairable) (Novák et al., 2003). In the case of unrepairable or severe damage, another pathway called Apoptosis becomes activated to direct the cell into a programmed cell death in order to prevent uncontrolled cell proliferation that may cause cancer (Roos \& Kaina, 2006).

The activity of family of proteins called Cyclindependent kinases (Cdks) is known as the main controller of mammalian cell cycle regulation. To become active and functional, Cdks need to be bound to a family of regulatory sub-units called cyclins. There are four main cyclins in mammalian cell cycle (Cyclin D, Cyclin E, Cyclin A, and Cyclin B) that affect the cell cycle progression in different stages (Figure 1). In fact, it is periodic synthesis and degradation of these cyclins that regulates Cdks activity in a timely manner (Malumbres \& Barbacid, 2009). Therefore, study of regulation of cyclins is a primary key to understanding the cell cycle control mechanism. Although there are other controlling mechanisms that impact the activity of Cdks or Cyclin/Cdks (see (Morgan, 2007) for details), the focus of this study is to characterize the dynamics of cyclins during the course of cell cycle.

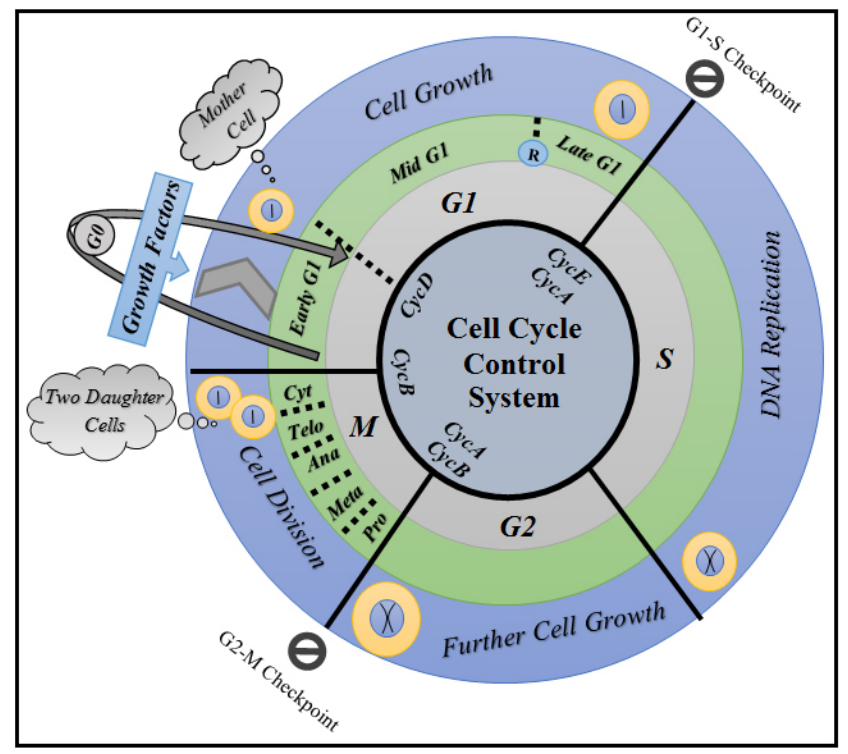

Figure 1. A holistic illustration of mammalian cell cycle.

To model the cell cycle dynamics, four main modelling approaches have been used: discrete, deterministic continuous, stochastic continuous, and hybrid. A collection of these modelling approaches can be found in a review by Csikász-Nagy (2009). Petri Net (PN) is a graphical tool that enables modelers to use a set of graphical elements to model and analyze biological systems in an intuitive manner. Thanks to its varied extensions, such as Discrete PN, Continuous PN, Stochastic PN, Hybrid PN, etc., it is possible to model complex systems at different levels of abstraction from high level to low level (Chaouiya, 2007). For instance, Mura and CsikászNagy (2008) presented a stochastic extension of PN for yeast cell cycle regulation and Fujita et al. (2004) developed a hybrid PN for fission yeast cell cycle which combined discrete and continuous schemes. However, there is no PN model for mammalian cell cycle regulation.

In current literature, mammalian cell cycle has been less investigated due to its complexity compared to other species, such as yeast. Therefore, there is a need to study and model this system in an intuitive yet comprehensive way so that even readers with less mathematical knowledge can understand the underlying mechanisms. The idea of developing such a model is partly inspired by the work of Singhania et al. (2011), 
who developed a simple hybrid model of Cyclin E, A and B regulations to simulate the flow cytometry data of cyclin concentrations in an asynchronous population of mammalian cell cycle. However, this model lacks Cyclin D, which is a vital cyclin during G1 phase. Furthermore, the Boolean part of this model does not have a proper updating scheme. Therefore, in our paper, we address these issues from two different points of view. From biological point of view, we include Cyclin D (and its regulators) in order to present a full picture of dynamic behavior of all cyclins (Cyclin D, E, A, and B) as the most important regulators of cell cycle from early G1 until the end of M phase. On the other hand, from modelling point of view, we use a hybrid paradigm incorporating discrete (for fast biochemical reactions, such as activation/inactivation) and deterministic continuous (for slow reactions, such as synthesis/degradation) modelling schemes. Furthermore, we adopt PN approach to present this hybrid modelling in the most intuitive way. And to further extend our hybrid PN in terms of granularity, multi-level extension is implemented and added to hybrid PN scheme. Thus, this MultiLevel Hybrid Petri Net (MLHPN) model is able to describe the whole mammalian cell cycle control system from cyclins point of view at three different levels (high, low, and functional levels). At high level of abstraction, the focus is on cyclins as one of the main controllers of the system. At low level, the regulators of the cyclins are investigated. And the focus of functional level is the corresponding function of cyclins in the transition between different cell cycle stages. The rest of this paper is organized as follows. The details of the proposed model are presented in Section 2 (in five sub-sections) and results and discussion are described in Section 3.

\section{MULTI-LEVEL HYBRID PETRI NET (MLHPN) MODEL}

\subsection{Biological Significance}

Cyclins are the core components of mammalian cell cycle control system and the study of their temporal dynamics is a key to better understanding cell cycle progression as well as phase transition. Note that since cyclins should bind to Cdks to form a functional component, whenever we talk about the effect of cyclins on other species, the indirect effect through this complex is considered. As shown in Figure 2, following the presence of growth factor, G0-G1 transcription factor (c-Myc) becomes activated (through growth factor signaling pathway) and stimulates the synthesis of Cyclin D. Initiation of Cyclin D production is an indication of G0-G1 transition and cell cycle initiation (Adhikary \& Eilers, 2005).

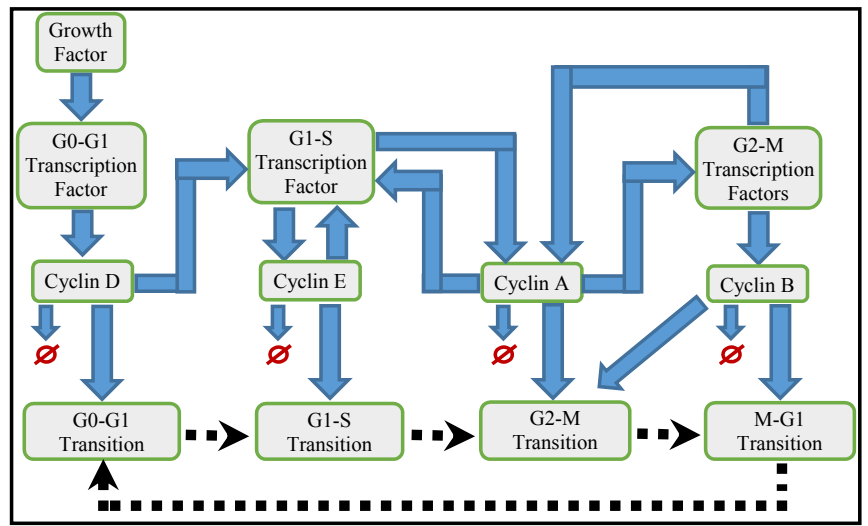

Figure 2. Important cyclins of mammalian cell cycle, their synthesis and degradation, and the corresponding effects on ordered sequence of cell cycle transitions.

Cyclin D (in complex with Cdk4\&6), in turn, stimulates production of Cyclin E (next cyclin in the series of cyclins) through activation of G1-S transcription factor (E2F). Cyclin E (in complex with Cdk2) forms a positive feedback loop with G1-S transcription factor that induces production of more Cyclin E that eventually results in G1-S transition (Figure 2) (Ji \& Dyson, 2010).

G1-S transcription factor also induces synthesis of next Cyclin, Cyclin A. It is the production of Cyclin A through G1-S transcription factor and G2-M transcription factors (b-Myb and NFY) that triggers the next critical event in cell cycle system which is G2-M transition (Chae \& Shin, 2011; Joaquin \& Watson, 2003). Cyclin A (in complex with Cdk2), also, helps the synthesis of Cyclin B through its positive feedback loop through G2-M transcription factors (Figure 2). Cyclin B has a key role during M phase and particularly in late $\mathrm{M}$ phase where its degradation through two ubiquitin ligases (APC/Cdc20 and APC/Cdh1) triggers the M-G1 transition event or exit from cell cycle. It is important to note that SCF ubiquitin ligase degrades Cyclin D and Cyclin E, while APC ubiquitin ligases degrade all cyclins excluding Cyclin E (Nakayama \& Nakayama, 2005; Peters, 2002). It should be also noticed that synthesis and degradation of cyclins are slow reactions, whereas activation and inactivation of regulators of cyclins (transcription factors and ubiquitin ligases) are by far faster. Therefore, in MLHPN model, cyclins and their synthesis/degradation are modelled continuously, while the regulation of their regulators is modelled discretely. 
Abroudi and Samarasinghe, System Modelling of Mammalian Cell Cycle Regulation Using Multi-Level Hybrid Petri Nets

\subsection{Key Elements of the Model}

As a Petri Net-based model, MLHPN comprises four key elements: places, transitions, arcs, and marking. Places represent species (such as proteins), while transitions indicate biochemical reactions. Arcs, on the other hand, denote the type of interaction between species and carry numbers (thresholds) that correspond to stoichiometric coefficients. Marking is used to represent the value of places. If a place is discrete, the marking is in the form of tokens, whereas, the marking for a continuous place is expressed as a real number. A list of elements utilized in MLHPN model is shown in Table 1. Discrete Delayed transitions can be fired after a predefined delay if the markings of their pre-places (the places that enter into a transition) are more than the corresponding arcs' thresholds. However, continuous transitions can be fired continuously as long as the marking of their pre-places is non-zero. Macro-elements are used for multi-level presentation of the system. The main feature of macro-elements is that they appear at high level of abstraction, while the elements inside them appear at low level. Generally, a macroplace represents a place-bounded set of elements, whereas a macro-transition signifies a set of transition-bounded elements. Logical elements are also utilized to represent a particular node (place or transition) in different reactions in a model and they are represented in gray color (see Table 1). Standard arcs denote regular biochemical reactions with corresponding Table 1. The elements used in the model.

stoichiometric coefficients. Read arcs are used to represent reactions in which the marking is not consumed, such as enzymatic reactions, and a transition with an inhibitory arc can take place only if the marking of the corresponding pre-place is less than the arc's threshold (Blätke et al., 2011). For a detailed description of Petri Nets, the reader is referred to a book written by (Koch et al., 2010).

The software used to develop the model is Snoopy (Heiner et al., 2012). A simple example of water formation using Petri Nets is given in Figure 3 where one molecule of Oxygen and two molecules of Hydrogen are required to produce two molecules of water, discretely. As seen in Figure 3, the firing rule for this transition is $m 1 \geq 1$ and $m 2 \geq 2$. Therefore, if the firing rule is satisfied, the reaction takes place and the next marking of places is calculated by integration of all the corresponding input (with negative sign) and output (with negative sign) arcs (Figure 3).

$1 \mathrm{O}_{2}+2 \mathrm{H}_{2} \rightarrow 2 \mathrm{H}_{2} \mathrm{O}$

Firing rule: $m 1 \geq 1$ and $m 2 \geq 2$

Initial Marking: $\{m 1=5, m 2=4, m 3=0\}$

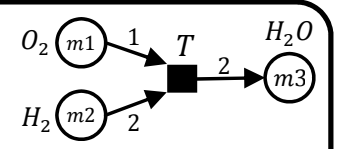

Next marking: $m 1=5-1=4, m 2=4-2=2, m 3=0+2=2$

Figure 3. Modelling of water formation using Petri Nets.

\subsection{High Level View of MLHPN}

The high level view of MLHPN presents the big picture of the model in which the control system is based on an ordered sequence of cyclins synthesis and degradation (Figure 4). As shown in Figure 4, following the presence of growth factor, first, the growth factor macro-place triggers the production of $\mathrm{CycD}$ through continuous transition T1. Then, CycD interacts with a macro-transition called G1_S_TF_Activation (the details of the elements inside each macro elements are presented in Section 2.5). This macro-transition, in turn, stimulates the synthesis of $\mathrm{CycE}$ and $\mathrm{CycA}$. Then, CycA interacts with the last macro-transition (G2_M_TF_Activation) which encompasses a set of elements that results in synthesis of $\mathrm{CycB} \& \mathrm{CycA}$ itself. The degradation of cyclins, on the other hand, is mediated through macro-places UBQ_D, UBQ_E, UBQ_A, and UBQ_B (Figure 4).

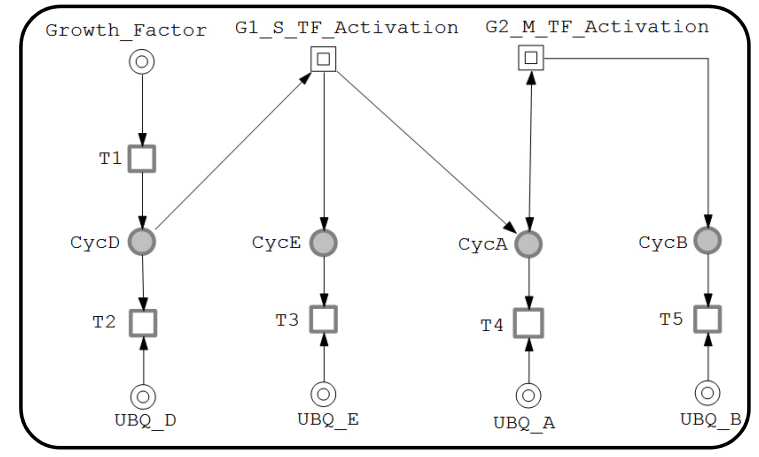

Figure 4. High level view of MLHPN model.

\subsection{Functional Level View of MLHPN}

At the Functional level, the function of different cyclins in mammalian cell cycle progression is modelled. The important stages in mammalian cell cycle (Early_G1, Mid_G1, Late_G1, S, G2, Prophase, Metaphase, Anaphase, and Telophase) are modelled as discrete places (as shown in Figure 5). The transition between 
Abroudi and Samarasinghe, System Modelling of Mammalian Cell Cycle Regulation Using Multi-Level Hybrid Petri Nets

events are either controlled by the level of cyclins or take place after some specific delays. The required levels and delays are partly adopted from a paper published by Singhania et al. (2011) in which a flow cytometry data of cyclin levels has been utilized for model development. It should be noted that in MLHPN, time and concentrations have arbitrary units and each cell cycle, which is approximately 24 hours, is equivalent to around 135 time-points in the model simulation.

If Early_G1 is assumed as the current state of the system, following the presence of growth factor, after 10 time-points delay, Sensing_GF transition (Figure 5) is fired and the state of the system is changed to Mid_G1. During this stage, $\mathrm{CycD}$ concentration increases. It is assumed that when the level of CycD passes a threshold (12 units of concentration), R_Point transition is fired and G1-S transcription factor becomes activated and stimulates synthesis of some target proteins including CycE \& CycA. Therefore, upon passing a threshold concentration, these cyclins trigger G1_S transition which alters the system state from Late_G1 to S (Figure 5). For cell cycle system to progress from $S$ to $G 2$ phase, DNA_Rep transition should be fired and it happens 8 hours after G1_S transition. The next transition in this ordered sequence of events is G2_M transition whose firing is controlled by CycA and CycB. It is important to emphasize that the transitions between M phase events (Prophase, Metaphase, Anaphase, and Telophase) occur according to some pre-defined delays (Singhania et al., 2011). Although the effect of $\mathrm{CycB}$ on $\mathrm{G} 2 \mathrm{M}$ transition is mediated through a read arc, its effect on the last cell cycle transition (M_G1) is modelled by an inhibitory arc. The reason for this is that the first transition is fired if the level of $\mathrm{CycB}$ becomes higher than an upper threshold (15.6), while the latter takes place if the corresponding level falls down below a lower threshold (0.4).

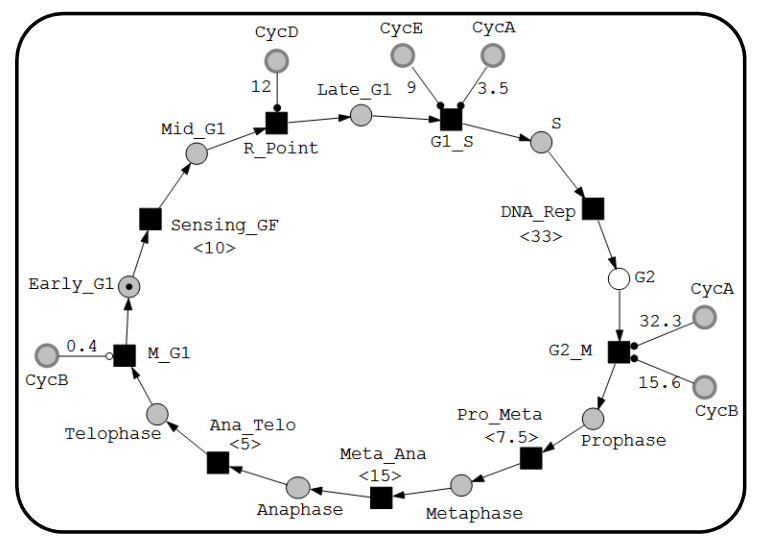

Figure 5. Functional level view of MLHPN model.

\subsection{Low Level View of MLHPN}

At low level of abstraction, the elements inside all macro places and transitions will be described. The corresponding elements of macro-places (Growth Factor, UBQ_D, UBQ_E, UBQ_A, and UBQ_B) are shown in Figure 6(A) to Figure 6(E). The low level elements of macro transitions G1_S_TF_Activation and G2_M_TF_Activation are presented in Figures 6(F) and 6(G). Furthermore, the rules for firing transitions, thresholds of arcs, initial values of places, and transition delays can be intuitively perceived from Figures 6 (initial values of all cyclins are assumed to be zero). Relevant continuous transition functions are presented in Table 2. With these equations, it is possible to represent the dynamics of cyclins (Figure 7).

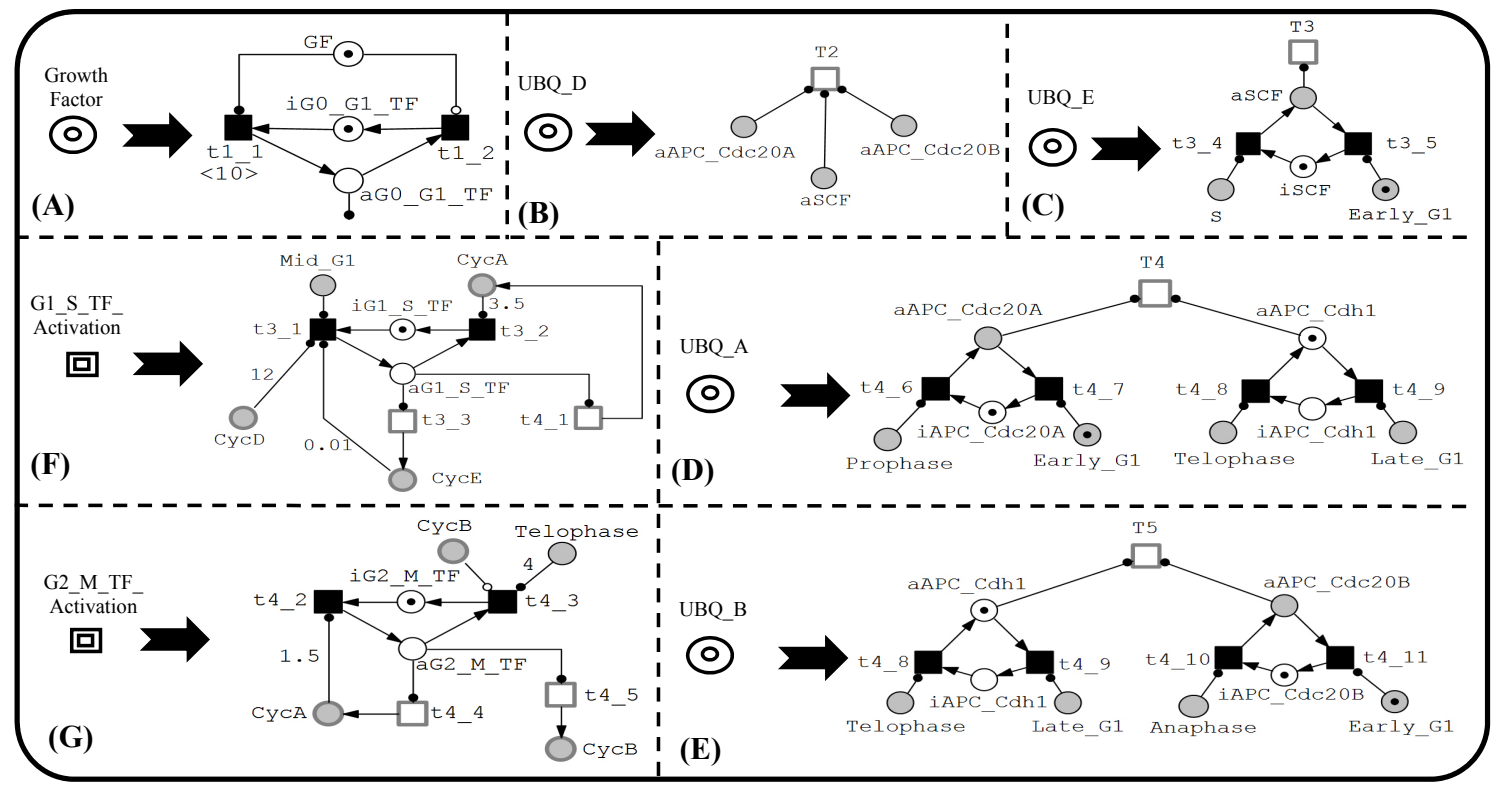

Figure 6. Low level view of MLHPN. The corresponding elements of macro-places and -transitions are represented from (A) to $(\mathrm{G})$. 
Abroudi and Samarasinghe, System Modelling of Mammalian Cell Cycle Regulation Using Multi-Level Hybrid Petri Nets

Table 2. Model equations for cyclin (continuous places).

\begin{tabular}{|c|c|}
\hline Cyclins & Equations \\
\hline Cyclin D & $\frac{d(C y c D)}{d t}=0.001+0.5 a G_{0-} G_{1-} T F-0.028 a S C F-0.5 a A P C_{-} C d c 20 A-0.3 a A P C_{-} C d c 20 B$ \\
\hline Cyclin E & $\frac{d(C y c E)}{d t}=0.005+0.5 a G_{1 \_} S_{-} T F-0.2 a S C F$ \\
\hline Cyclin A & $\frac{d(C y c A)}{d t}=0.005+0.1 a G_{1 \_} S_{-} T F+0.5 a G_{2-} M_{-} T F-0.15 a A P C_{-} C d c 20 A-0.8 a A P C_{-} C d h 1$ \\
\hline Cyclin B & $\frac{d(C y c B)}{d t}=0.005+0.25 a G_{2} M_{-} T F-0.1 a A P C_{-} C d c 20 B-0.8 a A P C_{-} C d h 1$ \\
\hline
\end{tabular}

\section{RESULTS AND DISCUSSION}

The importance of Cyclin D, as the initiator of cell cycle system as well as a therapeutic target in cancer, has been reported (Musgrove et al., 2011). According to detailed view of MLHPN (Figure 6), after 10 time-points of growth factor presence, inactive G0_G1_TF becomes activated and initiates synthesis of $\mathrm{CycD}$ and this transcription factor remains active as long as GF is available (Figure 6(A)). Now that the system state has been switched from early_G1 to Mid_G1 (Figure 5), CycD concentration starts to increase and the corresponding trend can be seen in model simulation in Figure 7. If this level crosses a threshold (12), transition t3_1 will be fired which leads to activation of $\mathrm{G} 1$ S_TF and thereby production of $\mathrm{CycE}$ and $\mathrm{Cyc} \overline{\mathrm{A}}$ through continuous transitions t3_3 and t4_1, respectively (Figures 6(F) \& 7). It should be noticed that when the level of CycA becomes higher than a threshold (3.5), this

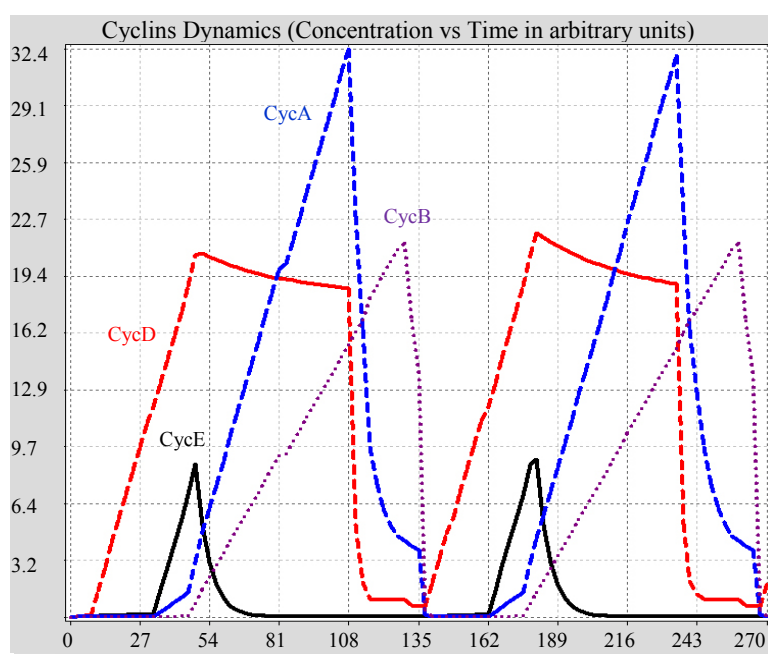

Figure 7. Temporal dynamics of cyclins during two cell cycles (each takes 135 time-points). cyclin stimulates inactivation of G1 S TF (Figures 6(F)). CycA, also, triggers the activation of G2_M_TF through transition t4_2 when its level crosses a threshold (1.5). Activated transcription factor, in turn, induces the production of $\mathrm{CycA}$ and $\mathrm{CycB}$ through continuous transition t4_4 and t4_5, respectively (Figure 6(G)). These dynamics can also be seen in model simulation in Figure 7.

In the case of cyclin degradation, as demonstrated in Figure 6(B), CycD is degraded by active SCF and APC_Cdc20 through continuous transition T2. But, degradation of CycE is just mediated by active SCF through continuous transition T3 (Figure $6(\mathrm{C})$ ). That is why a dramatic fall is seen in CycE level (G1_S transition) in Figure 7. APC_Cdc20 was assumed to have two different versions, APC_Cdc20A, and APC_Cdc20B (Figure 6(D \& E) ) as it has been reported that these ubiquitin ligases have more affinity to CycA and $\bar{C} y c B$, respectively (Morgan, 2007). Therefore, as illustrated in Figure 7, CycA starts to decrease (at the end of G2 phase) through ubiquitination by APC_Cdc20A but CycB level falls in the middle of M phase (Anaphase) where APC_Cdc20B becomes activated. However, APC_Cdh1, which becomes activated at the end of M phase (Telophase), degrades both cyclins and therefore end of Telophase is considered the end of cell cycle or M_G1 transition (Singhania et al., 2011).

In this paper, we addressed the issues of a hybrid model proposed by Singhania et al. (2011) through development of our model. Cyclin D and the corresponding regulators, which were missing in that model, were added into MLHPN model to track the temporal dynamics of all cyclins (Cyclin D, E, A, and B) throughout the cell cycle. We also introduced a novel approach to modelling the cell cycle by representing the system in three levels: high level, functional level, and low level. The high level presentation renders a big picture of the model (Figure 4). At the functional level, the function of cyclins in controlling transitions between different cell cycle stages was described (Figure 5). Finally, the details of regulators of cyclins were presented at low level (Figure 6). The simulation results based on simple mass action equations (Table 2) were illustrated in Figure 7 and are in good agreement with simulations and experimental data reported by Singhania et al. (2011). The significance of this study is mainly related to developing a minimal model that can represent the behavior of cyclins as key controllers of mammalian cell cycle. More importantly, the dynamic of cyclins (e.g. the peaktime) can be used as a biomarker for transitions between cell cycle phases and further investigation of this 
Abroudi and Samarasinghe, System Modelling of Mammalian Cell Cycle Regulation Using Multi-Level Hybrid Petri Nets

complex system. For example, the peak-time of Cyclin E has been used as a biomarker for G1-S transition (Ling et al., 2010). Furthermore, this paper sets the stage for the study of cell cycle arrest in the presence of DNA damage where different what-if scenarios can be applied to investigate the system response in various conditions, such as different levels of DNA damage. In future, other cell cycle regulators, such as CKIs and Tyrosine kinases \& phosphatases will also be incorporated to present a more comprehensive picture of mammalian cell cycle regulation.

\section{REFERENCES}

Adhikary, S., \& Eilers, M. (2005). Transcriptional regulation and transformation by Myc proteins. Nature Reviews Molecular Cell Biology, 6(8), 635-645.

Berridge, Michael J. (2012). Cell Signalling Biology: Module 9 - Cell Cycle and Proliferation. Biochemical Journal. doi:10.1042/csb0001009

Blätke, M., Heiner, M., \& Marwan, W. (2011). Tutorial-petri nets in systems biology. Otto von Guericke University and Magdeburg, Centre for Systems Biology.

Chae, H. D., \& Shin, D. Y. (2011). NF-Y binds to both G1-and G2-specific cyclin promoters; a possible role in linking CDK2/Cyclin A to CDK1/Cyclin B. Biochemistry and Molecurar Biology Reports, 44(8), 553-557.

Chaouiya, C. (2007). Petri net modelling of biological networks. Briefings in bioinformatics, 8(4), 210-219.

Csikász-Nagy, A. (2009). Computational systems biology of the cell cycle. Briefings in bioinformatics, 10(4), 424-434.

Fujita, S., Matsui, M., Matsuno, H., \& Miyano, S. (2004). Modeling and simulation of fission yeast cell cycle on hybrid functional Petri net. IEICE Transactions on Fundamentals of Electronics, Communications and Computer Sciences, 87(11), 2919-2928.

Heiner, M., Herajy, M., Liu, F., Rohr, C., \& Schwarick, M. (2012). Snoopy-a unifying Petri net tool. In Application and Theory of Petri Nets (pp. 398-407): Springer.

Ji, J.-Y., \& Dyson, N. J. (2010). Interplay between Cyclin-dependent Kinases and E2F-dependent Transcription. In Cell Cycle Deregulation in Cancer (pp. 23-41): Springer.

Joaquin, M., \& Watson, R. (2003). Cell cycle regulation by the B-Myb transcription factor. Cellular and Molecular Life Sciences CMLS, 60(11), 2389-2401.

Koch, I., Reisig, W., \& Schreiber, F. (2010). Modeling in systems biology: the Petri net approach (Vol. 16): Springer Science \& Business Media.

Ling, H., Kulasiri, D., \& Samarasinghe, S. (2010). Robustness of G1/S checkpoint pathways in cell cycle regulation based on probability of DNA-damaged cells passing as healthy cells. Biosystems, 101(3), 213-221.

Malumbres, M., \& Barbacid, M. (2009). Cell cycle, CDKs and cancer: a changing paradigm. Nature Reviews Cancer, 9(3), 153-166.

Morgan, D. O. (2007). The cell cycle: principles of control. London: New Science Press.

Mura, I., \& Csikász-Nagy, A. (2008). Stochastic Petri Net extension of a yeast cell cycle model. Journal of theoretical biology, 254(4), 850-860.

Musgrove, E. A., Caldon, C. E., Barraclough, J., Stone, A., \& Sutherland, R. L. (2011). Cyclin D as a therapeutic target in cancer. Nature Reviews Cancer, 11(8), 558-572.

Nakayama, K. I., \& Nakayama, K. (2005). Regulation of the cell cycle by SCF-type ubiquitin ligasesElsevier. Symposium conducted at the meeting of the Seminars in cell \& developmental biology

Novák, B., Sible, J. C., \& Tyson, J. J. (2003). Checkpoints in the cell cycle. eLS, 1-8.

Peters, J.-M. (2002). The anaphase-promoting complex: proteolysis in mitosis and beyond. Molecular cell, 9(5), 931-943.

Roos, W. P., \& Kaina, B. (2006). DNA damage-induced cell death by apoptosis. Trends in molecular medicine, $12(9), 440-450$.

Singhania, R., Sramkoski, R. M., Jacobberger, J. W., \& Tyson, J. J. (2011). A hybrid model of mammalian cell cycle regulation. PLoS computational biology, 7(2), e1001077.

Zetterberg, A., Larsson, O., \& Wiman, K. G. (1995). What is the restriction point? Current opinion in cell biology, 7(6), 835-842. 